Revue d'histoire de l'Amérique française

DAV REVE D.HISTOIRE DE L'AMÉRIQUE FRANÇAISE

\title{
Démographie différentielle en Nouvelle-France : villes et campagnes
}

\author{
Lorraine Gadoury, Yves Landry et Hubert Charbonneau
}

Volume 38, numéro 3, hiver 1985

Population et histoire

URI : https://id.erudit.org/iderudit/304283ar

DOI : https://doi.org/10.7202/304283ar

Aller au sommaire du numéro

Éditeur(s)

Institut d'histoire de l'Amérique française

ISSN

0035-2357 (imprimé)

1492-1383 (numérique)

Découvrir la revue

Citer cet article

Gadoury, L., Landry, Y. \& Charbonneau, H. (1985). Démographie différentielle en Nouvelle-France : villes et campagnes. Revue d'histoire de l'Amérique

française, 38(3), 357-378. https://doi.org/10.7202/304283ar d'utilisation que vous pouvez consulter en ligne.

https://apropos.erudit.org/fr/usagers/politique-dutilisation/ 


\title{
DÉMOGRAPHIE DIFFÉRENTIELLE EN NOUVELLE-FRANCE: VILLES ET CAMPAGNES*
}

\author{
LORRAINE GADOURY \\ YVES LANDRY \\ HUBERT CHARBONNEAU \\ Département de démographie \\ Université de Montréal
}

\section{Introduction}

La division d'une population en groupes et l'étude du comportement démographique distinct de chacun d'eux permettent de révéler des agissements spécifiques liés aux conditions de vie et à la position économique et sociale des individus. Cette répartition pose cependant le problème délicat de l'identification des sous-populations: doit-on, par exemple, mettre l'accent sur l'opposition ville-campagne ou encore sur la distinction entre groupes sociaux?

Un essai de réponse a été donné par Pierre Chaunu, qui croit que «les comportements sont plus géographiques que sociaux, plus communautaires qu'économiques» et qu' «il faut un fort gradient de différenciation sociale pour obtenir une différence appréciable de comportement devant la vie» ${ }^{1}$. Jacques Dupâquier a repris cette idée en soutenant que «le poids des antagonismes sociaux sur le destin démographique des populations n'était sans doute pas le même au XVII et au XVIII qu' au XIX ${ }^{\mathrm{e}}$ siècles [et qu'] il est peu probable que les situations sociales se reflètent intégralement dans les comportements démographiques». D'après lui, «l'opposition entre villes et campagnes n'est pas niable $[\ldots]$ mais elle est aussi géographique que sociale»"

En effet, on sait que les citadins français ont, sous l'Ancien Régime, un comportement démographique distinct de celui des ruraux, encore

\footnotetext{
* Ce texte a pu être préparé grâce à l'appui financier du Conseil de recherches en sciences humaines du Canada, du Fonds FCÁC et de l'Université de Montréal. Les auteurs expriment leurs remerciements à Marc Tremblay.

1 Pierre Chaunu, «Réflexions sur la démographie normande», dans Sur la population française au XVIII et au XIX $X^{e}$ siècles. Hommage à Marcel Reinhard (Paris, Société de démographie historique, 1973), 109 et 114.

2 Jacques Dupâquier, «Histoire et démographie», Population, 32, no spécial (septembre 1977): 310 .
} 
que les différences soient plus ou moins accentuées selon les régions et la taille des villes ${ }^{3}$. Les caractères les plus marqués sont une plus forte proportion de célibataires dans les villes, un âge au premier mariage plus élevé et une proportion également plus élevée de naissances illégitimes et de conceptions prénuptiales. De plus, en période prémalthusienne, le recours à la mise en nourrice rend les femmes urbaines plus fécondes que celles de la campagne (la durée de l'allaitement étant écourtée); la natalité reste cependant plus faible en ville en raison de la moindre durée des unions. Enfin, les habitants des villes ont tendance à délaisser les rythmes dictés par la terre, de sorte que les fluctuations saisonnières des mariages et des conceptions y présentent moins d'ampleur qu'à la campagne ${ }^{4}$.

Ces caractéristiques propres peuvent donc être dues en partie au facteur géographique, c'est-à-dire à l'entassement de la population dans un espace restreint, de forte densité et souvent malsain où, de plus, la mobilité de la population est très forte. Cependant, cet élément géogra-

TABLEAU 1

Distribution des familles suivant la profession du mari

\begin{tabular}{|c|c|c|c|c|c|}
\hline Catégorie & $\begin{array}{l}\text { Nombre } \\
\text { absolu }\end{array}$ & $\%$ & Classe & $\begin{array}{l}\text { Nombre } \\
\text { absolu }\end{array}$ & $\%$ \\
\hline $\begin{array}{l}\text { 1. Seigneurs et } \\
\text { officiers militaires }\end{array}$ & 48 & 7,0 & supérieure & 107 & 15,6 \\
\hline $\begin{array}{l}\text { 2. Marchands et } \\
\text { fonctionnaires }\end{array}$ & 59 & 8,6 & & & \\
\hline $\begin{array}{l}\text { 3. Gens de métier } \\
\text { 4. Habitants }\end{array}$ & $\begin{array}{r}88 \\
331\end{array}$ & $\begin{array}{l}12,8 \\
48,1\end{array}$ & moyenne & 419 & 60,9 \\
\hline $\begin{array}{l}\text { 5. Voyageurs et } \\
\text { cabaretiers } \\
\text { 6. Soldats, journaliers } \\
\text { et domestiques }\end{array}$ & 54 & 7,8 & inférieure & 83 & 12,0 \\
\hline 7. Indéterminée & 79 & 11,5 & indéterminée & 79 & 11,5 \\
\hline Total & 688 & 100,0 & & 688 & 100,0 \\
\hline
\end{tabular}

3 Jean-Pierre Bardet, «La démographie des villes de la modernité (XVI ${ }^{\mathrm{e}}$-XVIII ${ }^{\mathrm{e}}$ siècles): mythes et réalités», Annales de démographie historique (1974): 117-124.

4 Ernest Labrousse et al., Histoire économique et sociale de la France, tome II: 1660-1789 (Paris, PUF, 1970), 72; Jacques Dupâquier, La population française aux XVII et XVIII siècles (Paris, PUF, 1979), 54, 60-61; Jean-Pierre Bardet, op. cit., 117-124. 
phique est indissociable du facteur social; en effet, on ne peut nier que les villes recouvrent une entité sociale différente et plus diversifiée que celle des campagnes: pas d'agriculteurs mais la majorité des membres des classes supérieures de la société (marchands, notables, administrateurs...), avec la présence de divers types d'artisans, de domestiques, etc. On peut donc penser, avec Alfred Perrenoud, que la spécificité urbaine passe par le canal des structures sociales et que «chaque ensemble, chaque strate révèle des particularités que l'analyse globale tend à confondre» 5 .

Un nouveau problème se pose cependant à qui veut diviser une population en groupes sociaux. En effet, quel critère retenir pour définir des régimes démographiques spécifiques? Jusqu'à présent, les études françaises ont basé leurs divisions sur la pratique endogamique ${ }^{6}$, sur le niveau de fortune ${ }^{7}$, le statut professionnel ${ }^{8}$, la division sociogéographique $^{9}$ ou encore sur des critères plus subjectifs ${ }^{10}$.

Pour la Nouvelle-France, les deux principales études démographiques, celles de Jacques Henripin ${ }^{11}$ et d'Hubert Charbonneau ${ }^{12}$, considèrent la population dans son ensemble et n'opèrent pas de découpage socio-économique. Par contre, deux travaux récents ont porté sur des catégories socio-professionnelles particulières, celle des voyageurs vers les Pays-d'en-Haut ${ }^{13}$ et des marchands de Montréal ${ }^{14}$.

De plus, Yves Landry et Hubert Charbonneau, dans le texte d'une communication présentée à Bucarest en 1980 et basée sur le même échantillon que Vie et mort de nos ancêtres, ont étudié le comportement

5 Alfred Perrenoud, «Variables sociales en démographie urbaine. L'exemple de Genève au XVIII ${ }^{\mathrm{e}}$ siècle», dans Démographie urbaine $X V^{e}-X X^{e}$ siècle (Université Lyon II, 1977): 171.

6 N.W. Mogensen, «Structures et changements démographiques dans vingt paroisses normandes sous l'Ancien Régime. Une analyse sociale», Annales de démographie historique (1975): 343-367.

J.-C. Giacchetti et M. Tyvaert, «Argenteuil (1740-1790)», Annales de démographie historique (1969), 40-61

8 Hubert Charbonneau, Tourouvre-au-Perche aux XVII et XVIII siècles. Étude de démographie historique (Paris, PUF, 1970), 423 p.; Alfred Perrenoud, op . cit., 143-172 et «L'inégalité sociale devant la mort à Genève au XVII ${ }^{\mathrm{e}}$ siècle», Population, $30^{\mathrm{e}}$ année, no spécial (novembre 1975): 221-243; Alain Bideau, La châtellenie de Thoissey-en-Dombes (1650-1840): étude d'histoire démographique - analyse différentielle des phénomènes démographiques, Thèse pour le doctorat de troisième cycle (Centre Pierre Léon, Université Lyon II, 1980), 684 p. dact.

9 Jean-Claude Perrot, Genèse d'une ville moderne, Caen au XVIII ${ }^{e}$ siècle (Paris, Mouton, 1973), $1157 \mathrm{p}$. $310 \mathrm{p}$

Pierre Guillaume, La population de Bordeaux au XIX ${ }^{e}$ siècle (Paris, A. Colin, 1972),

Ii Jacques Henripin, La population canadienne au début du XVIII siècle: nuptialité-féconditémortalité infantile (Paris, PUF, 1954), 129 p.

${ }_{12}$ Hubert Charbonneau, Vie et mort de nos ancêtres. Étude démographique (Montréal, Les Presses de l'Université de Montréal, 1975), 268 p.

${ }_{13}$ Hubert Charbonneau, Bertrand Desjardins et Pierre Beauchamp, «Le comportement démographique des voyageurs sous le régime français», Histoire Sociale/Social History, XI, 21 (mai 1978): 120-133.

14 José Igartua, «Le comportement démographique des marchands de Montréal vers 1760», RHAF, 33, 3 (décembre 1979): 427-445. 
démographique différentiel des Canadiens selon leurs professions, telles que fournies par les généalogies ${ }^{15}$. La distribution des familles selon la profession du mari est la suivante ${ }^{16}$ :

En conclusion de leur étude, les auteurs ont fait ressortir quelques différences entre les trois classes: premièrement, les mariages sont d'autant plus précoces, tant chez les hommes que chez les femmes, que l'on descend dans l'échelle sociale; en second lieu, la durée de l'union est plus courte dans la classe inférieure ce qui, additionné à l'effet de l'âge au mariage, produit un âge moyen au veuvage plus précoce, indice d'une certaine surmortalité dans les couches les plus pauvres de la société. La fécondité est plus forte dans les familles de la classe supérieure qui, profitant d'une plus longue durée d'union, comptent donc un plus grand nombre d'enfants; enfin, les conceptions prénuptiales sont plus rares dans cette classe que dans les deux autres. Dans une autre communication portant sur la mortalité différentielle des enfants ${ }^{17}$, les mêmes auteurs ont démontré que le taux de mortalité infantile de la classe inférieure était nettement au-dessus de la moyenne.

Ces premières études ont donc montré que le comportement démographique variait en fonction du statut socio-professionnel et des conditions matérielles sous-jacentes. Mais elles indiquent aussi qu'au Canada, en général, le destin démographique pesait d'un poids qui, sans être uniforme d'un groupe social à l'autre, n'avait pas le caractère discordant qu'on a pu constater ailleurs.

Dans la foulée de Vie et mort de nos ancêtres et des deux études de démographie différentielle qui ont suivi, nous avons opéré une autre division de la population de la Nouvelle-France, en séparant celle des villes de celle des campagnes. Cette division se justifie d'abord par le fait que, comparativement à la France d'Ancien Régime, rurale à plus de $85 \%{ }^{18}$, le Canada au tournant du XVIII' siècle se distingue par l'importance relative de ses villes, Québec, Trois-Rivières et Montréal; en effet, on y retrouve un peu plus d'un Canadien sur quatre ${ }^{19}$.

15 Yves Landry et Hubert Charbonneau, «Démographie différentielle et catégories sociales en Nouvelle-France», dans Actes duXVe Congrès international des sciences historiques [Bucarest] (Éditura Academiei Republicii Socialiste România, 1982), IV: 1150-1163.

${ }_{16}$ Le fait qu'il ait été convenu, pour cette classification, de retenir la profession la plus élevée de l'homme en ayant exercé plusieurs, a pu gonfler les résultats des catégories supérieures et ainsi atténuer les éventuelles différences de comportement démographique.

17 Hubert Charbonneau et Yves Landry, «La mortalité différentielle des enfants issus des mariages célébrés au Canada au XVII ${ }^{\mathrm{e}}$ siècle», communication présentée au Congrès général de l'Union internationale pour l'étude scientifique de la population, Manille (Philippines) (décembre 1981).

18 Pierre Goubert, L'Ancien Régime, tome I: La Société (Paris, A. Colin, 1969): 165

19 Guy Frégault, «La Nouvelle-France, territoire et population», dans Le XVIII ${ }^{e}$ siècle canadien. Études (Montréal, HIMH, 1970), 38. Frégault se base, pour avancer ce chiffre, sur les recensements de 1698, 1706, 1707 et 1713. Pour sa part, Marcel Trudel évalue à environ un tiers la proportion de la population qui, en 1663, vivait sans aucune base rurale; La population du Canada en 1663 (Montréal, Fides, 1973), 21. 
Il est certain que le nombre absolu d'habitants de ces trois villes à cette époque a été très faible: environ 2000 pour Québec, autant pour Montréal et 300 pour Trois-Rivières. Cependant, le fait important, c'est que les administrateurs de la colonie ainsi que les habitants les décrivent comme «villes» et qu'elles sont perçues dès le début comme te ${ }^{20}$. C'est ainsi qu'en 1742, l'évêque de Québec, Mgr de Pontbriand, met l'accent sur les fonctions sociales que remplissent les villes: éducation, assistance, administration et défense territoriale, afin de convaincre les ruraux d'aller y porter du blé malgré la disette.

Les villes sont, Nos Très Chers Enfants, comme le centre de cette colonie; deviendriez-vous des membres injustes qui refuseraient au coeur attaqué un sang qui lui est nécessaire? ${ }^{21}$

Est-ce que, malgré leur population peu élevée, les villes de la Nouvelle-France étaient assez différentes de la campagne environnante pour engendrer des traits démographiques distincts? À quoi seraient dues ces éventuelles différences? Voilà les questions auxquelles nous tenterons de répondre ici, à partir d'une nouvelle répartition des fiches de famille ayant servi à Hubert Charbonneau dans l'ouvrage Vie et mort de nos ancêtres.

Nous exposerons dans une première partie nos sources et décrirons brièvement les sous-populations qui feront l'objet de comparaisons. Par la suite, nous en étudierons la nuptialité, la fécondité et la mortalité des enfants, ce qui nous permettra, en conclusion, de faire ressortir les traits distinctifs des urbains et des ruraux du Canada des XVII ${ }^{\mathrm{e}}$ et $\mathrm{XVIII}{ }^{\mathrm{e}}$ siècles.

\section{Données de base et description des sous-populations}

\section{Le répertoire de Godbout}

Les données sur lesquelles se fonde cette étude proviennent principalement du répertoire généalogique et bio-bibliographique d'Archange Godbout ${ }^{22}$. Celui-ci a rédigé des commentaires biographiques sur tous les immigrants, ainsi que sur leurs fils et petits-fils, ayant fondé une famille dans la colonie avant 1700. Comme la méthode utilisée par Godbout permet d'avoir une vision longitudinale (les individus sont suivis depuis leur naissance jusqu'à leur décès), son ouvrage est d'un grand intérêt pour la démographie historique.

20 Louise Dechêne remarque que «ceux qui fondèrent et habitèrent Montréal ne désignèrent jamais autrement que par le mot «ville» l'espace de 110 arpents réservé à cette fin vers 1648 et assez tôt délimité par une palissade»; «La croissance de Montréal au XVIII ${ }^{\mathrm{e}}$ siècle», RHAF, 27, 2 (septembre 1973): 165.

21 Guy Frégault, op. cit., 40.

22 Archange Godbout, «Nos ancêtres au XVII ${ }^{\mathrm{e}}$ siècle», RAPQ (1951-53): 447-544; (195355): 443-536; (1955-57): 377-489; (1957-59): 381-440; (1959-60): 275-354; (1965): 145-181. 
Cependant, l'oeuvre de Godbout est restée inachevée. En effet, seules les notices des familles dont les noms commencent par A et B (jusqu'à Brassard inclusivement) ont été publiées. Cet échantillon de la population canadienne (environ 15\%) est cependant, pour le démographe, suffisant pour généraliser les résultats de son analyse à l'échelle de la population dans son entier. D'ailleurs, la méthode d'échantillonnage consistant à ne retenir que les noms de famille débutant par une lettre précise a déjà été utilisée avec succès en démographie historique ${ }^{23}$.

Il faut également souligner que l'oeuvre de Godbout est d'essence généalogique et ne se destine aucunement à l'exploitation à des fins démographiques. Ainsi, son auteur a eu tendance à laisser de côté des familles n'ayant pas eu de descendance, de même que des individus restés célibataires ou encore des enfants morts en bas âge. Hubert Charbonneau a expliqué ailleurs comment, des 585 fiches de famille tirées directement du répertoire de Godbout, il a dû en rajouter 103 autres puisées dans le Dictionnaire Tanguay ${ }^{24}$. Celui-ci a d'ailleurs servi, avec les recensements, à trouver les dates de naissance des épouses dont les noms ne commençaient pas par A ou $\mathrm{B}^{25}$.

La population étudiée étant quasi fermée, on devrait connaitre toutes les dates de naissance, mariage et décès de tous les enfants de la plupart des familles. Il en va autrement parce que la valeur des données dont nous disposons dépend étroitement de la qualité de l'enregistrement qui en a été fait à l'époque. Charbonneau a déjà observé que le sousenregistrement a surtout affecté les sépultures dans une proportion qui approche $25 \%{ }^{26}$. Heureusement, on possède différents moyens pour combler les lacunes des registres, ainsi qu'on le verra lors de l'étude de la fécondité et de la mortalité des enfants.

\section{Distribution de la population}

Archange Godbout ne s'est pas limité dans son répertoire à relever les dates de naissance, mariage et décès des individus; il fournit également des renseignements sur leur résidence et leurs activités, à partir des répertoires des greffes de notaires, recensements et autres sources.

\footnotetext{
23 Jean-Pierre Bardet, loc. cit., 110.

24 Cyprien Tanguay, Dictionnaire généalogique des familles canadiennes (Montréal, Eusèbe Senécal, 1871-1890), 7 volumes.

${ }_{25}$ On pourra se reporter à Vie et mort de nos ancêtres, op. cit., 49-60, pour une critique approfondie de l'oeuvre de Godbout et des détails sur les corrections apportées à l'échantillon initial. Il est à remarquer que les résultats présentés ici diffèrent quelquefois de ceux donnés dans Vie et mort... même si le matériau de base est le même. C'est que les fiches de famille contiennent des renseignements sur les parents qui ont fondé des familles avant 1700 ainsi que sur leurs enfants nés pour la plupart au XVIII ${ }^{\mathrm{e}}$ siècle. Alors que Charbonneau s'est concentré surtout sur les enfants, nous nous attarderons ici surtout sur les parents pour lesquels il était possible de déterminer l'habitat; nous n'avons utilisé les données relatives aux enfants que pour l'étude des célibataires et celle de la mortalité infantile.

26 Ibid, 69-114.
} 
C'est ce qui a permis de distribuer les 688 fiches de famille exploitées en trois groupes distincts: les urbains, les ruraux et les mixtes; le pourcentage de chaque groupe par rapport à l'ensemble est représenté au tableau 2.

TABLEAU 2

Distribution des familles suivant l'habitat

\begin{tabular}{ccccc}
\hline Urbain & Rural & Mixte & Indéterminé & Ensemble \\
\hline 125 & 473 & 85 & 5 & 688 \\
$18,2 \%$ & $68,8 \%$ & $12,3 \%$ & $0,7 \%$ & $100 \%$ \\
\hline
\end{tabular}

Sont considérées comme urbaines les familles pour lesquelles le mariage du couple et la naissance des enfants ont eu lieu dans une des trois villes de la colonie et qui semblent y avoir résidé tout au long de leur union. Les familles rurales sont celles pour lesquelles le mariage et la naissance des enfants sont enregistrés ailleurs que dans les trois villes et qui habitaient donc à la campagne ${ }^{27}$. Enfin, sont classées comme mixtes les familles qui ont vécu les deux situations, c'est-à-dire qui ont passé plusieurs années de leur vie de couple à la ville et plusieurs à la campagne. Comme nous le verrons par la suite, ces familles migrantes ont un comportement démographique spécifique, ce qui interdit de les ranger de façon arbitraire avec les urbains ou les ruraux.

Il est intéressant de noter qu'en distribuant les familles mixtes de façon égale entre les urbains et les ruraux (ce qui devrait refléter la situation observée à une date précise), on obtient un pourcentage de $24 \%$ d'urbains. Cette proportion est conforme à l'évaluation avancée par Frégault et vient donc confirmer la valeur du classement que nous avons effectué.

\section{Caractéristiques des sous-populations}

Avant de passer à l'étude des traits démographiques des trois souspopulations, il convient d'en donner quelques caractéristiques générales.

Premièrement, comme on pouvait s'y attendre, les activités économiques des urbains et des ruraux diffèrent grandement. Le tableau 3 distribue les familles selon la profession du mari, classée suivant la grille du tableau 1.

Ce tableau permet de faire ressortir le caractère principalement agricole des ruraux: 60\% d'entre eux sont d'abord des exploitants de la

27 Les paroisses situées en banlieue de Québec et sur l'île de Montréal mais en dehors des limites de la ville sont considérées comme rurales. 
terre et ce pourcentage passe à $69 \%$ si l'on retranche du total les $13 \%$ d'individus de profession indéterminée. Les urbains occupent des fonctions plus reliées au caractère même de la ville: ils sont gens de métier (30\%), marchands et fonctionnaires $(22 \%)$, seigneurs et officiers militaires (16\%); $13 \%$ d'entre eux seulement sont classés comme exploitants agricoles.

TABLEAU 3

Distribution des familles suivant l'habitat et la profession du mari*

\begin{tabular}{|c|c|c|c|c|c|c|c|c|}
\hline \multirow{2}{*}{$\begin{array}{c}\text { Profession } \\
\text { du mari }\end{array}$} & \multicolumn{2}{|c|}{ Urbain } & \multicolumn{2}{|c|}{ Rural } & \multicolumn{2}{|c|}{ Mixte } & \multicolumn{2}{|c|}{ Ensemble } \\
\hline & $\begin{array}{c}\text { Nombre } \\
\text { absolu }\end{array}$ & $\%$ & $\begin{array}{c}\text { Nombre } \\
\text { absolu }\end{array}$ & $\%$ & $\begin{array}{c}\text { Nombre } \\
\text { absolu }\end{array}$ & $\%$ & $\begin{array}{c}\text { Nombre } \\
\text { absolu }\end{array}$ & $\%$ \\
\hline $\begin{array}{l}\text { 1. Seigneurs } \\
\text { et } \\
\text { officiers } \\
\text { militaires }\end{array}$ & 20 & $(16,0)$ & 25 & $(5,3)$ & 3 & $(3,5)$ & 48 & $(7,0)$ \\
\hline $\begin{array}{l}\text { 2. Marchands } \\
\text { et fonc- } \\
\text { tionnaires }\end{array}$ & 27 & $(21,6)$ & 18 & $(3,8)$ & 12 & $(14,1)$ & 57 & $(8,4)$ \\
\hline $\begin{array}{l}\text { 3. Gens de } \\
\text { métier }\end{array}$ & 37 & $(29,6)$ & 34 & $(7,2)$ & 16 & $(18,8)$ & 87 & $(12,7)$ \\
\hline 4. Habitants & 16 & $(12,8)$ & 284 & $(60,0)$ & 32 & $(37,6)$ & 332 & $(48,6)$ \\
\hline $\begin{array}{l}\text { 5. Voyageurs } \\
\text { et caba- } \\
\text { retiers }\end{array}$ & 11 & $(8,8)$ & 35 & $(7,4)$ & 6 & $(7,1)$ & 52 & $\cdot(7,6)$ \\
\hline $\begin{array}{l}\text { 6. Soldats, } \\
\text { journaliers } \\
\text { et domes- } \\
\text { tiques }\end{array}$ & 5 & $(4,0)$ & 14 & $(3,0)$ & 10 & $(11,8)$ & 29 & $(4,3)$ \\
\hline $\begin{array}{l}\text { 7. Indéter- } \\
\text { minée }\end{array}$ & 9 & $(7,2)$ & 63 & $(13,3)$ & 6 & $(7,1)$ & 78 & $(11,4)$ \\
\hline Total & 125 & $(100,0)$ & 473 & $(100,0)$ & 85 & $(100,0)$ & 683 & $(100,0)$ \\
\hline
\end{tabular}

* Ne sont pas compris dans ce tableau ni dans les suivants les 5 familles d'habitat indéterminé du tableau 2 .

Les occupations reliées aux familles mixtes présentent un caractère hétérogène, ce qui n'étonne en rien: $38 \%$ d'habitants, ainsi qu'une proportion relativement importante de gens de métier (19\%), de marchands et fonctionnaires ( $14 \%$ ) et de soldats, journaliers et domestiques $(12 \%)$. Il ne semble donc pas que le changement de type d'habitat soit réservé aux plus démunis, même si c'est chez les mixtes que l'on retrouve la plus forte proportion d'occupations des catégories 5 et 6 , prises ensemble. 
D'autres caractéristiques procèdent de la division des familles suivant l'origine combinée des conjoints: immigrants ou Canadiens (tableau $4)$.

TABLEAU 4

Distribution des familles suivant l'habitat et l'origine combinée des conjoints

\begin{tabular}{lccccccccc}
\hline \multirow{2}{*}{$\begin{array}{l}\text { Origine des } \\
\text { conjoints }\end{array}$} & \multicolumn{2}{c}{ Urbain } & \multicolumn{2}{c}{ Rural } & \multicolumn{2}{c}{ Mixte } & \multicolumn{2}{c}{ Ensemble } \\
\cline { 2 - 10 } & $\begin{array}{c}\text { Nombre } \\
\text { absolu }\end{array}$ & $\%$ & $\begin{array}{c}\text { Nombre } \\
\text { absolu }\end{array}$ & $\%$ & $\begin{array}{c}\text { Nombre } \\
\text { absolul }\end{array}$ & $\%$ & $\begin{array}{c}\text { Nombre } \\
\text { absolu }\end{array}$ & $\%$ \\
\hline $\begin{array}{l}\text { Français- } \\
\text { Française }\end{array}$ & 44 & $(35,2)$ & 143 & $(30,2)$ & 34 & $(40,0)$ & 221 & $(32,4)$ \\
$\begin{array}{l}\text { Français- } \\
\text { Canadienne }\end{array}$ & 49 & $(39,2)$ & 151 & $(32,0)$ & 36 & $(42,3)$ & 236 & $(34,6)$ \\
$\begin{array}{l}\text { Canadien- } \\
\text { Française }\end{array}$ & 2 & $(1,6)$ & 3 & $(0,0)$ & 1 & $(1,2)$ & 6 & $(0,8)$ \\
$\begin{array}{l}\text { Canadien- } \\
\text { Canadienne }\end{array}$ & 30 & $(24,0)$ & 176 & $(37,2)$ & 14 & $(16,5)$ & 220 & $(32,2)$ \\
\hline \begin{tabular}{l} 
Total \\
\hline
\end{tabular} & 125 & $(100,0)$ & 473 & $(100,0)$ & 85 & $(100,0)$ & 683 & $(100,0)$ \\
\hline
\end{tabular}

On remarque d'abord que les couples formés de deux Canadiens sont plus fréquents chez les ruraux que chez les deux autres groupes. Les familles d'habitat mixte constituent à cet égard un groupe vraiment à part: les couples de Canadiens y sont très peu nombreux et 5 familles sur 6 mettent en présence au moins un conjoint immigrant.

L'étude de la distribution des familles selon la période du mariage (tableau 5) permet de mieux expliquer ces différences.

TABLEAU 5

Distribution des familles suivant l'habitat et la période de mariage

\begin{tabular}{lccccccccc}
\hline & \multicolumn{2}{c}{ Urbain } & \multicolumn{2}{c}{ Rural } & \multicolumn{2}{c}{ Mixte } & \multicolumn{2}{c}{ Ensemble } \\
\cline { 2 - 11 } $\begin{array}{l}\text { Période de } \\
\text { mariage }\end{array}$ & $\begin{array}{c}\text { Nombre } \\
\text { absolu }\end{array}$ & $\begin{array}{c}\text { Nombre } \\
\text { absolu }\end{array}$ & $\%$ & $\begin{array}{c}\text { Nombre } \\
\text { absolu }\end{array}$ & $\%$ & $\begin{array}{c}\text { Nombre } \\
\text { absolu }\end{array}$ & $\%$ \\
\hline $\begin{array}{l}\text { Avant 1680 } \\
\begin{array}{l}1680 \text { et } \\
\text { après }\end{array}\end{array}$ & 52 & $(41,6)$ & 177 & $(37,4)$ & 45 & $(52,9)$ & 274 & $(40,1)$ \\
\hline Total & 73 & $(58,4)$ & 296 & $(62,6)$ & 40 & $(47,1)$ & 409 & $(59,9)$ \\
\hline
\end{tabular}


La catégorie mixte se distingue par sa proportion plus grande que la moyenne de couples formés avant 1680 , ce qui s'accorde bien avec le fait qu'on y retrouve plus de conjoints immigrants que dans les deux autres sous-catégories. A l'opposé, les ruraux ont le plus fort pourcentage d'unions contractées après 1680, alors qu'on y décèle le plus de mariages entre Canadiens.

\section{Nuptialité}

\section{Pourcentage de célibataires}

L'évaluation du pourcentage de célibataires, qui révèle l'intensité de la nuptialité, est particulièrement intéressante lorsqu'on compare les populations urbaine et rurale, puisqu'on peut s'attendre à retrouver de grands écarts entre les indices; en effet, les célibataires comme les religieux et religieuses sont habituellement concentrés à la ville.

La fréquence du célibat est obtenue ici en calculant la proportion de célibataires parmi les hommes et les femmes décédés à 50 ans et plus, âge où le célibat est probablement définitif.

TABLEAU 6

État matrinnonial des Canadiens décédés à 50 ans et plus suivant le sexe et l'habitat

\begin{tabular}{|c|c|c|c|c|c|c|c|c|c|c|}
\hline \multirow[b]{3}{*}{ Habitat } & \multicolumn{5}{|c|}{ Hommes } & \multicolumn{5}{|c|}{ Femmes } \\
\hline & \multirow{2}{*}{$\begin{array}{c}\text { Mariés } \\
\text { et } \\
\text { veufs }\end{array}$} & \multicolumn{2}{|c|}{ Célibataires } & \multirow{2}{*}{$\begin{array}{l}\text { Indé- } \\
\text { ter- } \\
\text { miné }\end{array}$} & \multirow[t]{2}{*}{ Total } & \multirow{2}{*}{$\begin{array}{c}\text { Mariées } \\
\text { et } \\
\text { veuves }\end{array}$} & \multicolumn{2}{|c|}{ Célibataires } & \multirow{2}{*}{$\begin{array}{l}\text { Indé- } \\
\text { ter- } \\
\text { miné }\end{array}$} & \multirow[t]{2}{*}{ Total } \\
\hline & & $\begin{array}{l}\text { Non re- } \\
\text { ligieux }\end{array}$ & $\begin{array}{l}\text { Reli- } \\
\text { gieux }\end{array}$ & & & & $\begin{array}{l}\text { Non re- } \\
\text { ligieu- } \\
\text { ses }\end{array}$ & $\begin{array}{c}\text { Reli- } \\
\text { gieu- } \\
\text { ses }\end{array}$ & & \\
\hline Urbain & $\begin{array}{c}51 \\
(86,4)\end{array}$ & $\begin{array}{c}6 \\
(10,2)\end{array}$ & $\begin{array}{c}2 \\
(3,4)\end{array}$ & $\begin{array}{c}0 \\
(0,0)\end{array}$ & $\begin{array}{c}59 \\
(100)\end{array}$ & $\begin{array}{c}56 \\
(70,9)\end{array}$ & $\begin{array}{c}3 \\
(3,8)\end{array}$ & $\begin{array}{c}15 \\
(19,0)\end{array}$ & $\begin{array}{c}5 \\
(6,3)\end{array}$ & $\begin{array}{r}79 \\
(100)\end{array}$ \\
\hline Rural & $\begin{array}{c}395 \\
(95,4)\end{array}$ & $\begin{array}{c}12 \\
(2,9)\end{array}$ & $\begin{array}{c}2 \\
(0,5)\end{array}$ & $\begin{array}{c}5 \\
(1,2)\end{array}$ & $\begin{array}{c}414 \\
(100)\end{array}$ & $\begin{array}{c}304 \\
(93,0)\end{array}$ & $\begin{array}{c}12 \\
(3,7)\end{array}$ & $\begin{array}{c}7 \\
(2,1)\end{array}$ & $\begin{array}{c}4 \\
(1,2)\end{array}$ & $\begin{array}{c}327 \\
(100)\end{array}$ \\
\hline Mixte & $\begin{array}{c}58 \\
(92,1)\end{array}$ & $\begin{array}{c}5 \\
(7,9)\end{array}$ & $\begin{array}{c}0 \\
(0,0)\end{array}$ & $\begin{array}{c}0 \\
(0,0)\end{array}$ & $\begin{array}{c}63 \\
(100)\end{array}$ & $\begin{array}{c}58 \\
(85,2)\end{array}$ & $\begin{array}{c}5 \\
(7,4)\end{array}$ & $\begin{array}{c}5 \\
(7,4)\end{array}$ & $\begin{array}{c}0 \\
(0,0)\end{array}$ & $\begin{array}{c}68 \\
(100)\end{array}$ \\
\hline Ensemble & $\begin{array}{c}504 \\
(94,0)\end{array}$ & $\begin{array}{c}23 \\
(4,3)\end{array}$ & $\begin{array}{c}4 \\
(0,8)\end{array}$ & $\begin{array}{c}5 \\
(0,9)\end{array}$ & $\begin{array}{c}536 \\
(100)\end{array}$ & $\begin{array}{c}418 \\
(88,2)\end{array}$ & $\begin{array}{c}20 \\
(4,2)\end{array}$ & $\begin{array}{l}27 \\
(5,7)\end{array}$ & $\begin{array}{c}9 \\
(1,9)\end{array}$ & $\begin{array}{c}474 \\
(100)\end{array}$ \\
\hline
\end{tabular}

Les résultats présentés au tableau 6 confirment nos attentes: les différences sont en effet très marquées entre les sous-populations, puisque le célibat des urbains (religieux et non religieux) est quatre fois plus élevé que celui des ruraux. Notons cependant que le célibat des hommes est rarement dû à l'entrée en religion, les communautés canadiennes faisant venir leurs prêtres de France. Par contre, on constate un 
nombre relativement élevé de religieuses dans la population urbaine. Comme celles-ci proviennent surtout de familles aisées ${ }^{28}$, on peut comprendre que le pourcentage soit plus fort à la ville puisque c'est là, on l'a vu au tableau 3, que se retrouvent le plus de familles ayant des fonctions importantes. De plus, les maisons d'enseignement et les institutions religieuses sont également concentrées en milieu urbain.

\section{Âge au premier mariage et durée d' union}

Si l'on observe maintenant l'âge au premier mariage (tableau 7), on s'aperçoit que, là encore, la différence est significative: on se marie plus tard chez les urbains que chez les ruraux. Phénomène déjà observé pour la France, le mariage tardif des urbains pourrait s'expliquer par l'incidence des migrations qui retardent la formation des familles ${ }^{29}$.

TABLEAU 7

Âge moyen au premier mariage suivant le sexe et l'habitat

\begin{tabular}{lcccc}
\hline Sexe & Urbain & Rural & Mixte & Ensemble \\
\hline Époux & 29,7 & 28,1 & 28,7 & 28,4 \\
Épouses & 20,0 & 18,9 & 19,1 & 19,1 \\
\hline Écart & 9,7 & 9,2 & 9,6 & 9,3 \\
\hline
\end{tabular}

L'étude de la durée moyenne des unions révèle également des éléments de différenciation (tableau 8).

TABLEAU 8

Durée moyenne des unions suivant l'habitat

\begin{tabular}{cccc}
\hline Urbain & Rural & Mixte & Ensemble \\
\hline 17,9 & 23,6 & 28,7 & 23,2 \\
\hline
\end{tabular}

L'écart entre urbains et ruraux laisserait croire à une mortalité plus forte à la ville qu'à la campagne, ce qui était sans doute le cas comme nous le verrons plus loin. La durée d'union dans la catégorie mixte est quant à elle beaucoup plus longue que celle des deux autres: 11 ans de plus que les urbains et 5 de plus que les ruraux! Cette apparente vitalité des migrants s'explique cependant, au moins en partie, par la définition même de la catégorie: celle-ci est en effet formée de couples qu'on a

28 Hubert Charbonneau, Vie et mort de nos ancêtres, op. cit., 170.

29 Jacques Dapâquier, La population française, op . cit., 60-61. Alfred Perrenoud, op . cit., 147. Pour sa part, Jean-Claude Perrot avance que, dans les villes, «l'intérêt personnel ou conjugal l'emporterait sur le projet purement familial et fécond», compte rendu de Jean-Pierre Bardet, Annales de démographie historique (1976): 472. 
pu observer pour des périodes de temps significatives à la ville et à la campagne. Quand l'union était rompue précocement, les conjoints n'avaient souvent pas eu le temps de changer d'habitat.

\section{Mouvement mensuel des mariages}

Il convient de clore l'étude différentielle de la nuptialité par l'examen du mouvement mensuel des mariages (tableau 9 et graphique 1).

TABLEAU 9

Mouvement mensuel des mariages suivant l'habitat

\begin{tabular}{|c|c|c|c|c|c|c|c|c|c|c|c|c|c|}
\hline & \multicolumn{13}{|c|}{ MOIS } \\
\hline Habitat & $\mathrm{J}$ & $\mathrm{F}$ & M & A & $\mathrm{M}$ & $\mathbf{J}$ & $\mathbf{J}$ & A & $\mathrm{S}$ & $\mathrm{O}$ & $\mathrm{N}$ & $\mathrm{D}$ & Total \\
\hline \multicolumn{14}{|l|}{ Urbain } \\
\hline nombre absolu & 16 & 9 & 2 & 7 & 6 & 9 & 6 & 8 & 10 & 17 & 28 & 4 & 122 \\
\hline nombre relatif* & 153 & 96 & 19 & 70 & 58 & 90 & 58 & 77 & 99 & 163 & 278 & 39 & 1200 \\
\hline \multicolumn{14}{|l|}{ Rural } \\
\hline nombre absolu & 28 & 53 & 12 & 23 & 15 & 21 & 29 & 28 & 35 & 72 & 105 & 17 & 438 \\
\hline nombre relatif* & 74 & 156 & 32 & 64 & 40 & 58 & 77 & 74 & 96 & 193 & 290 & 46 & 1200 \\
\hline \multicolumn{14}{|l|}{ Mixte } \\
\hline nombre absolu & 4 & 11 & 2 & 3 & 8 & 1 & 3 & 9 & 3 & 16 & 10 & 10 & 80 \\
\hline nombre relatif* & 59 & 178 & 29 & 46 & 118 & 15 & 44 & 133 & 46 & 235 & 151 & 146 & 1200 \\
\hline
\end{tabular}

* Cette répartition tient compte du nombre de jours dans chaque mois. En l'absence de variations saisonnières, il y aurait 100 mariages chaque mois pour un total de 1200 dans l'année.

Le graphique 1 montre que les comportements des urbains et des ruraux subissaient également l'influence du calendrier religieux: les creux de mars et décembre s'expliquent par l'interdiction des mariages pendant le carême et l'avent, et les pointes de février, octobre et novembre par l'imminence de ces périodes. Pour le reste, les deux courbes s'éloignent parfois (comme en janvier, février, mai, juin et juillet), mais ces différences ne semblent pas être significatives: le petit nombre de cas disponibles serait à la base de ces variations aléatoires ${ }^{30}$.

\section{Fécondité}

Pour l'étude de la fécondité, nous avons utilisé 415 familles $(61 \%$ du total), soit celles pour lesquelles on disposait des dates de début et de fin d'union.

30 Le mouvement mensuel de l'ensemble des mariages du XVII ${ }^{\mathrm{e}}$ siècle a déjà été analysé par Raymond Roy, Yves Landry et Hubert Charbonneau, «Quelques comportements des Canadiens au XVII ${ }^{e}$ siècle d'après les registres paroissiauX», RHAF, 31, 1 (juin 1977): 54-59. 


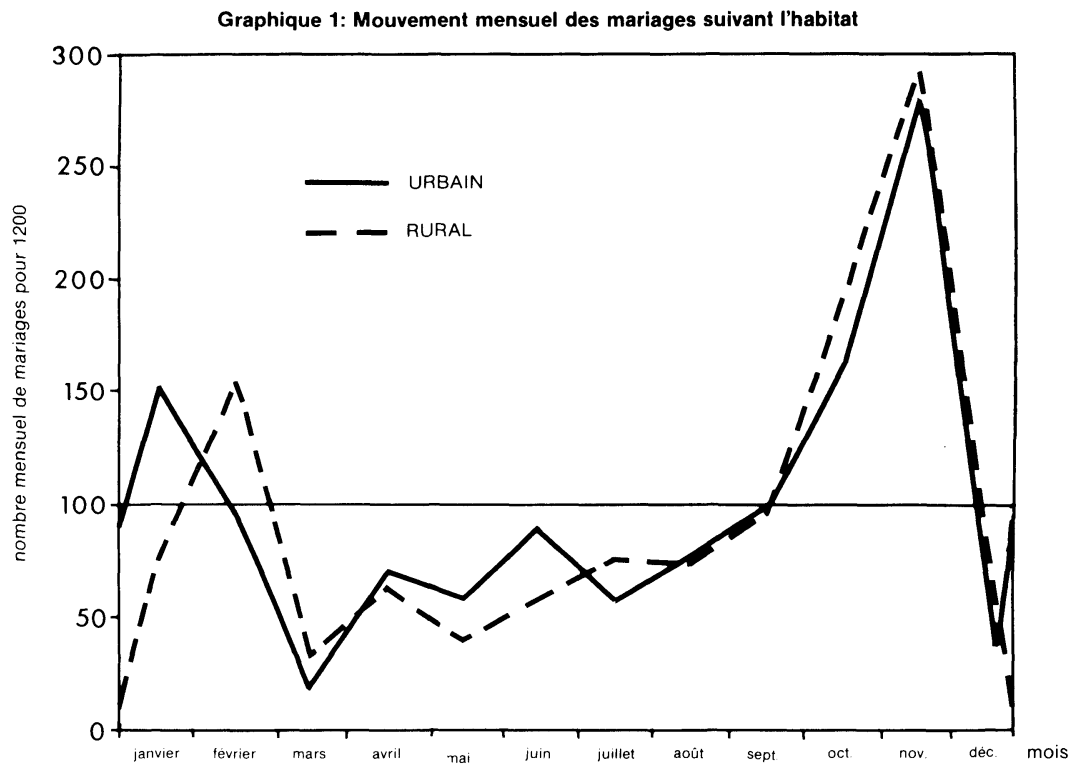

Source: Tableau 9

Taux de fécondité

On retrouve dans ces familles 2652 naissances «relevées», c'està-dire puisées dans les registres d'état civil. Elles ne forment cependant qu'une partie du total des naissances, puisque d'autres n'ont pu être relevées, soit à cause de la disparition des registres, soit parce qu'elles n'ont pas été enregistrées. Aussi convient-il, avant de calculer les taux de fécondité des femmes urbaines et rurales, d'effectuer les corrections nécessaires pour arriver à un nombre de naissances le plus près possible de la réalité.

Une première correction s'impose quant aux naissances «perdues», c'est-à-dire celles dont la disparition est liée au hasard (registres brûlés ou perdus). Pour évaluer leur nombre, nous avons appliqué la formule imaginée par Louis Henry, qui suppose que le rapport entre les mariages correspondant aux naissances «relevées» et ces naissances serait le même que celui entre les mariages correspondant aux naissances «perdues» et ces naissances ${ }^{31}$. Les 405 naissances perdues, qui se retrouvent comme on le constate au tableau 10 dans une proportion plus grande chez les ruraux où il $\mathrm{y}$ a eu plus de pertes de registres, ont

31 Cette formule est exposée dans Hubert Charbonneau, Vie et mort de nos ancêtres, op. cit., $72-73$. 
été ensuite réparties entre les groupes d'âges des femmes de la même façon que les naissances retrouvées par les chercheurs, puis ont été ajoutées aux naissances relevées.

Deux corrections supplémentaires sont cependant nécessaires. Elles concernent les ondoyés décédés avant 3 jours et à 3 jours et plus. La proportion des premiers devait être sous l'Ancien Régime, selon Louis Henry, de $3 \%{ }^{32}$; avant de l'appliquer à l'ensemble des naissances relevées et perdues, nous avons déduit de ce nombre le pourcentage d'ondoyés décédés déjà relevés dans les fiches de famille, puis nous avons ajouté $1 \%$ pour les enfants morts à 3 jours et plus sans avoir été baptisés ni mentionnés dans les registres. Nous avons ainsi ajouté 67 naissances, réparties entre urbains, ruraux et mixtes, tel qu'indiqué au tableau 10.

Les taux de fécondité légitime corrigés, présentés au tableau 11 et illustrés au graphique $2^{33}$, permettent de constater que les urbains ont une fécondité plus élevée que les ruraux, sauf après 40 ans. Cette plus forte fécondité de la population urbaine a déjà été observée pour la France $^{34}$ et tiendrait à deux facteurs: la mise en nourrice fréquente pour les urbains et la mortalité infantile plus élevée à la ville. Ces deux éléments réduisent la période de l'allaitement et permettent donc aux femmes urbaines de retrouver plus rapidement leur fertilité ${ }^{35}$.

TABLEAU 10

Distribution des naissances par catégorie suivant l'habitat

\begin{tabular}{|c|c|c|c|c|c|c|c|c|c|c|}
\hline \multirow[t]{2}{*}{ Habitat } & \multicolumn{2}{|c|}{$\begin{array}{c}\text { Naissances } \\
\text { relevées }\end{array}$} & \multicolumn{3}{|c|}{$\begin{array}{l}\text { Naissances } \\
\text { perdues } \\
\text { Avant } \\
3 \text { jours }\end{array}$} & \multicolumn{2}{|c|}{$\begin{array}{c}\text { Ondoyés } \\
\begin{array}{c}\text { À } 3 \text { jours } \\
\text { et plus }\end{array}\end{array}$} & & \multicolumn{2}{|c|}{$\begin{array}{l}\text { Naissances } \\
\text { totales }\end{array}$} \\
\hline & $\begin{array}{c}\text { Nbre } \\
\text { absolu }\end{array}$ & $\%$ & $\begin{array}{l}\text { Nbre } \\
\text { absolu }\end{array}$ & $\%$ & $\begin{array}{c}\text { Nbre } \\
\text { absolu }\end{array}$ & $\%$ & $\begin{array}{l}\text { Nbre } \\
\text { absolu }\end{array}$ & $\%$ & $\begin{array}{l}\text { Nbre } \\
\text { absolu }\end{array}$ & $\%$ \\
\hline Urbain & 428 & $(92,0)$ & 27 & $(5,8)$ & 5 & $(1,1)$ & 5 & $(1,1)$ & 465 & (100) \\
\hline Rural & 1836 & $(82,9)$ & 329 & $(14,9)$ & 28 & $(1,2)$ & 22 & $(1,0)$ & 2215 & (100) \\
\hline Mixte & 388 & $(87,4)$ & 49 & $(11,0)$ & 3 & $(0,7)$ & 4 & $(0,9)$ & 444 & (100) \\
\hline Ensemble & 2652 & $(84,9)$ & 405 & $(13,0)$ & 36 & $(1,1)$ & 31 & $(1,0)$ & 3124 & (100) \\
\hline
\end{tabular}

32 Ibid., 79.

33 Le taux de fécondité s'obtient en mettant en rapport le nombre de naissances par groupe d'âges avec le nombre d'années de mariage passées par les femmes à l'intérieur de ces groupes d'âges.

34 Jacques Dupâquier, La population française, op. cit., 54.

Sur la pratique de la mise en nourrice, voir Marie-France Morel, «Théories et pratiques de l'allaitement en France au XVIII ${ }^{\mathrm{e}}$ siècle», Annales de démographie historique (1976): 409410. Sur l'effet de l'allaitement en régime de fécondité naturelle, voir Fécondité naturelle: niveaux et déterminants de la fécondité naturelle, publié sous la direction de Henri Leridon et Jane Menken (Liège, Ordina Éditions, 1979), passim. 
TABLEAU 11

Taux corrigés de fécondité légitime suivant l'habitat (pour 1000 )

\begin{tabular}{|c|c|c|c|c|c|c|c|c|}
\hline \multirow[b]{2}{*}{ Habitat } & \multicolumn{8}{|c|}{ Groupes d'âges de la femme } \\
\hline & $\begin{array}{c}10-14 \\
\text { ans }\end{array}$ & $\begin{array}{c}15-19 \\
\text { ans }\end{array}$ & $\begin{array}{c}20-24 \\
\text { ans }\end{array}$ & $\begin{array}{c}25-29 \\
\text { ans }\end{array}$ & $\begin{array}{c}30-34 \\
\text { ans }\end{array}$ & $\begin{array}{c}35-39 \\
\text { ans }\end{array}$ & $\begin{array}{c}40-44 \\
\text { ans }\end{array}$ & $\begin{array}{c}45-49 \\
\text { ans }\end{array}$ \\
\hline Urbain & 102 & 470 & 539 & 489 & 463 & 449 & 208 & 0 \\
\hline Rural & 25 & 421 & 505 & 460 & 455 & 403 & 237 & 31 \\
\hline Mixte & 64 & 394 & 492 & 515 & 471 & 413 & 149 & 45 \\
\hline Ensemble & 45 & 423 & 510 & 473 & 460 & 413 & 221 & 29 \\
\hline
\end{tabular}

Graphique 2: Taux corrigés de fécondité légitime suivant l'habitat

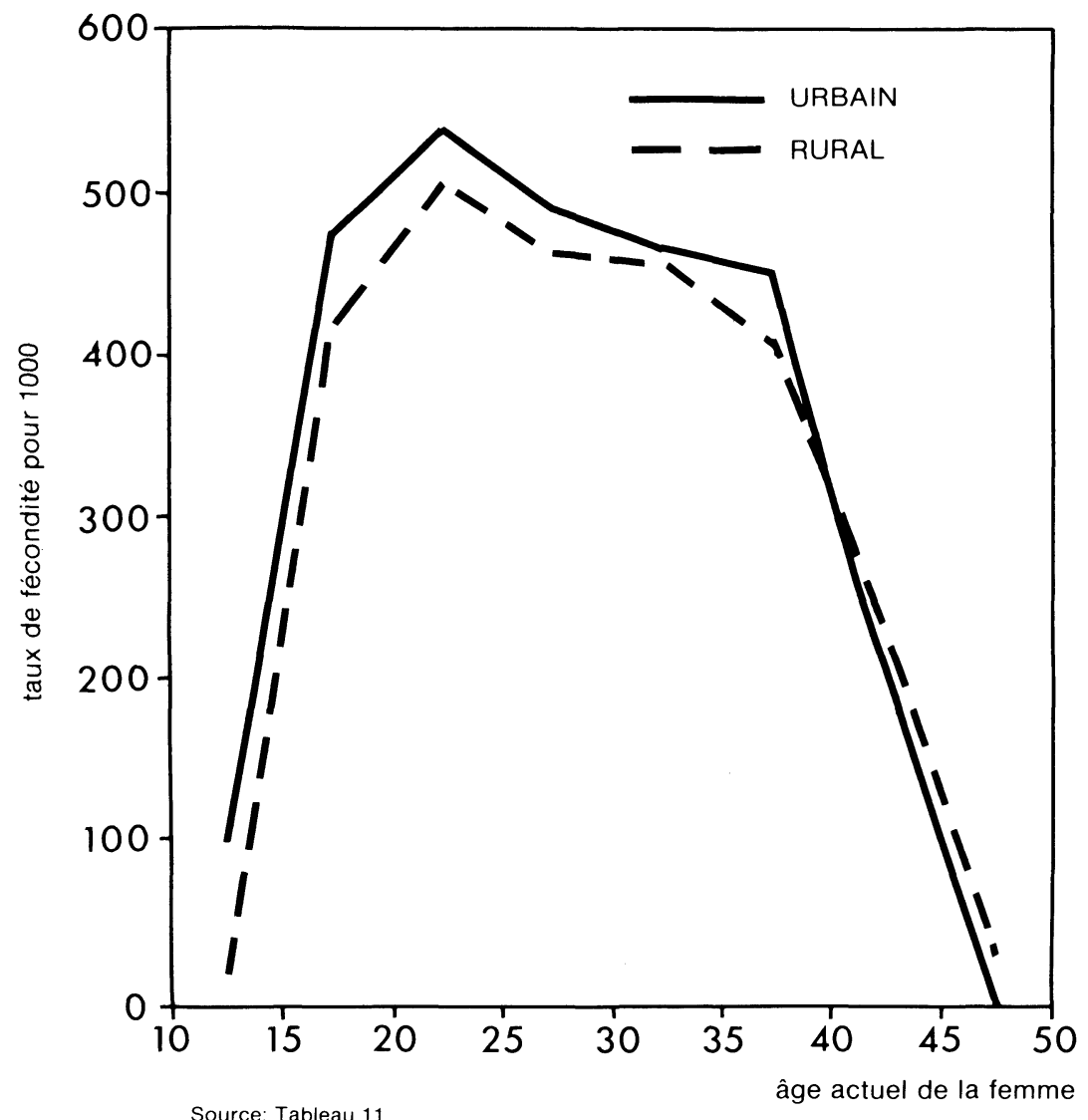




\section{Nombre d'enfants par famille}

Il n'y a pas nécessairement adéquation entre taux de fécondité et nombre moyen d'enfants et c'est ce que confirme le tableau 12. En effet, même avec les taux de fécondité les plus élevés, ce sont les urbains qui ont les familles les moins nombreuses (5,3 enfants en moyenne). De plus, la population mixte, avec des taux de fécondité se situant entre les urbains et les ruraux, se retrouve avec une moyenne d'enfants par famille très élevée: 8,2 enfants comparativement à 6,9 pour les ruraux ${ }^{36}$.

TABLEAU 12

Nombre moyen corrigé d'enfants par famille suivant l'habitat

\begin{tabular}{lccccccccc}
\hline & \multicolumn{2}{c}{ Urbain } & \multicolumn{2}{c}{ Rural } & \multicolumn{2}{c}{ Mixte } & \multicolumn{2}{c}{ Ensemble } \\
\cline { 2 - 8 } Familles & moyenne & nombre & moyenne & nombre & moyenne & nombre & moyenne nombre \\
\hline Complètes & 6,8 & 36 & 9,6 & 171 & 9,6 & 44 & 9,1 & 251 \\
Incomplètes & 4,6 & 82 & 5,9 & 251 & 6,4 & 36 & 5,6 & 369 \\
\hline Ensemble & 5,3 & 118 & 6,9 & 422 & 8,2 & 80 & 7,1 & 620 \\
\hline
\end{tabular}

Pour les comprendre, il faut mettre ces résultats en relation avec les données déjà exposées sur la nuptialité. Les tableaux 7 et 8 indiquent en effet que les urbains se mariaient en général plus tard que les ruraux et que la durée moyenne de leurs unions était plus courte. C'est donc cette période de fécondité réduite, due surtout à une plus grande mortalité à la ville, qui explique le nombre restreint d'enfants issus des familles urbaines. De la même façon, le nombre élevé d'enfants des familles mixtes découle de durées d'union relativement longues.

\section{Conceptions prénuptiales}

Résultat prévisible, les conceptions prénuptiales sont un peu plus fréquentes à la ville qu'à la campagne: $4,8 \%$ contre $3,4 \%$, à cause probablement d'un contrôle social moins strict en milieu urbain. La population mixte détient cependant le pourcentage le plus élevé: $6 \%$, et ce nombre doit être mis en rapport avec le fait que la catégorie mixte est formée surtout d'immigrants s'étant mariés assez tôt dans l'histoire de la colonie, quand l'encadrement social était moins fort.

36 Nous avons utilisé pour ce calcul l'ensemble des familles qu'on a pu déterminer comme complètes ou incomplètes et nous avons éliminé les autres. De plus, nous avons ajouté aux naissances contenues dans les fiches de famille le pourcentage des naissances inconnues par famille, soit $5 \%$ pour les urbains, $13 \%$ pour les ruraux et $11 \%$ pour les mixtes. 


\section{Âge de la mère à la dernière naissance}

L'âge moyen de la mère à la dernière naissance dépassait 40 ans à la ville comme à la campagne, que la femme se fût mariée précocement ou non (tableau 13). Cet âge élevé constitue une preuve de l'absence de limitation systématique des naissances.

TABLEAU 13

Âge moyen de la mère à la dernière naissance, suivant l'habitat

\begin{tabular}{lcccc}
\hline Âge au mariage de la mère & Urbain & Rural & Mixte & Ensemble \\
\hline Avant 20 ans & 42,9 & 40,6 & 40,1 & 40,6 \\
20 ans et plus & 40,5 & 40,6 & 41,8 & 40,8 \\
\hline
\end{tabular}

\section{Mouvement mensuel des naissances}

Contrairement à celui des mariages, le mouvement mensuel des naissances ne subit pas l'effet du calendrier religieux. Le lien existant entre les événements démographiques et les activités, ainsi que le genre de vie des individus, n'en ressort que davantage (tableau 14 et graphique 3 ).

Ainsi, pour la population rurale, le mouvement est assez clair: les conceptions culminent au début de l'été (mai et juin), puis baissent jusqu'à atteindre un creux en septembre et surtout en octobre, alors que les travaux des champs demandent le plus d'efforts. La remontée de novembre et décembre est due quant à elle aux nombreux mariages d'automne.

TABLEAU 14

Mouvement mensuel des naissances et conceptions suivant l'habitat

\begin{tabular}{|c|c|c|c|c|c|c|c|c|c|c|c|c|c|}
\hline \multirow{4}{*}{ Habitat } & \multicolumn{9}{|c|}{ Mois de naissance } & \multirow{3}{*}{$\mathrm{O}$} & \multirow{3}{*}{$\mathrm{N}$} & \multirow{3}{*}{ D } & \multirow{4}{*}{ Total } \\
\hline & $\mathrm{J}$ & $\mathrm{F}$ & $\mathrm{M}$ & A & $\mathrm{M}$ & $\mathrm{J}$ & $\mathrm{J}$ & A & $\mathrm{S}$ & & & & \\
\hline & \multicolumn{9}{|c|}{ Mois de conception } & & & & \\
\hline & A & M & $\mathrm{J}$ & $J$ & A & $\mathrm{S}$ & $\mathrm{O}$ & $\mathrm{N}$ & $\mathrm{D}$ & $\mathrm{J}$ & $\mathrm{F}$ & $\mathrm{M}$ & \\
\hline \multicolumn{14}{|l|}{ Urbain } \\
\hline nombre absolu & 49 & 39 & 56 & 53 & 49 & 55 & 46 & 51 & 48 & 48 & 34 & 56 & 584 \\
\hline nombre relatif* & 99 & 86 & 113 & 111 & 99 & 115 & 93 & 103 & 100 & 97 & 71 & 113 & 1200 \\
\hline \multicolumn{14}{|l|}{ Rural } \\
\hline nombre absolu & 223 & 260 & 243 & 230 & 232 & 202 & 172 & 222 & 205 & 193 & 214 & 193 & 2589 \\
\hline nombre relatif* & 101 & 129 & 110 & 108 & 105 & 95 & 78 & 101 & 96 & 88 & 101 & 88 & 1200 \\
\hline \multicolumn{14}{|l|}{ Mixte } \\
\hline nombre absolu & 46 & 47 & 46 & 42 & 44 & 39 & 42 & 46 & 42 & 48 & 43 & 51 & 536 \\
\hline nombre relatif* & 101 & 113 & 101 & 95 & 97 & 89 & 93 & 101 & 95 & 106 & 97 & 112 & 1200 \\
\hline
\end{tabular}

* Cette répartition tient compte du nombre de jours contenus dans chaque mois. En l'absence de variations saisonnières, il y aurait 100 naissances chaque mois pour un total de 1200 dans l'année. 


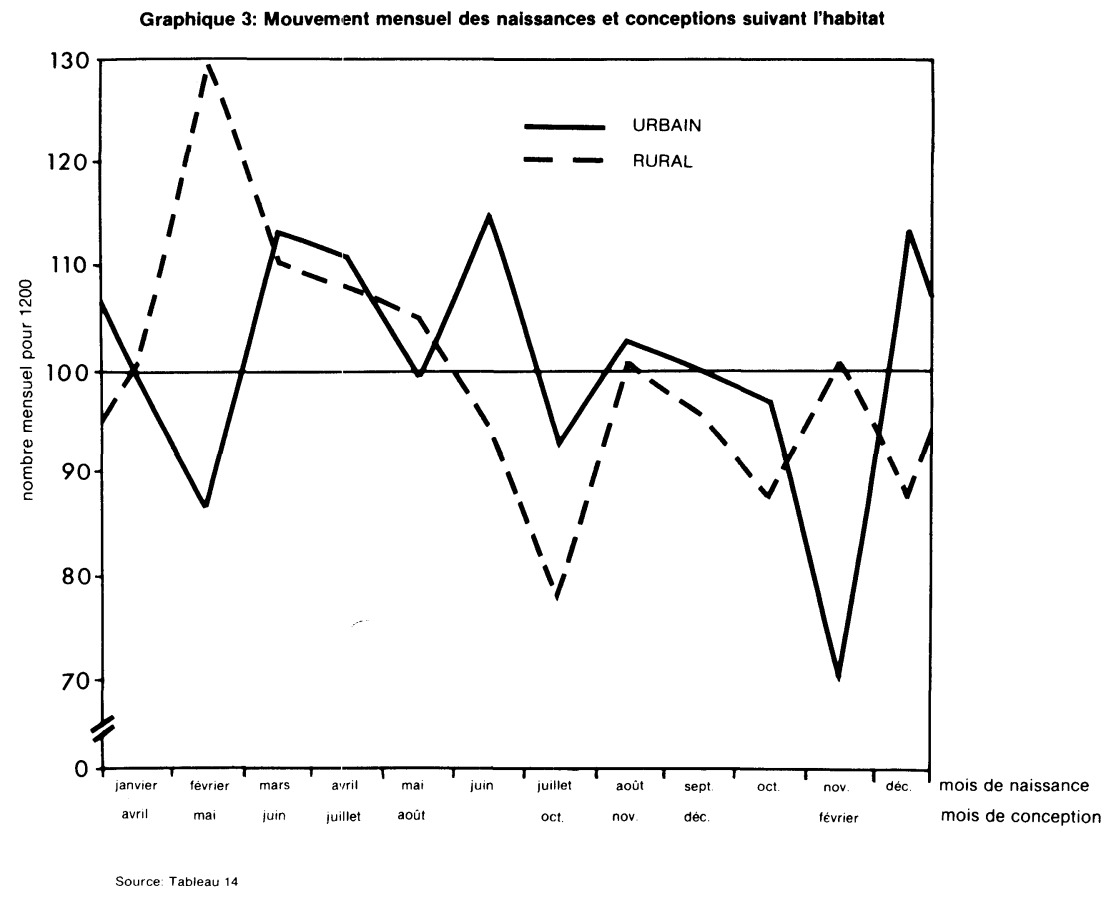

La courbe de la population urbaine est plus difficile à interpréter puisque les habitants des villes pratiquent plusieurs types d'activités ne commandant probablement pas un comportement commun, comme à la campagne. On peut remarquer quand même que les conceptions s'y font surtout à l'été (de juin à septembre) et que la baisse de l'automne est beaucoup moins marquée que chez les ruraux ${ }^{37}$.

\section{Mortalité des enfants}

Comme on l'a souligné plus haut, les lacunes dans l'enregistrement des sépultures, ainsi que les pertes de registres, ont été très importantes. Aussi faut-il apporter plusieurs corrections aux données originales avant de bâtir la table de mortalité des enfants.

Les 683 fiches de famille fournissent les dates de 858 décès d'enfants de moins de 15 ans; elles contiennent également 560 autres mentions d'enfants dont on ignore s'ils sont morts avant cet âge. Pour le déterminer, nous recourrons à la méthode des prénoms répétés ${ }^{38}$.

37 Raymond Roy, Yves Landry et Hubert Charbonneau, loc. cit., 59-60.

38 Exposée par Hubert Charbonneau, Vie et mort..., op. cit., 96-100. 
Cette méthode ingénieuse repose sur l'examen des prénoms que les parents donnaient à leurs enfants. On sait que le prénom d'un enfant décédé au seuil de la vie était souvent attribué au cadet, de sorte que la fréquence de cette répétition permet de calculer la proportion d'enfants de destin inconnu effectivement morts en bas âge. On en déduit ensuite l'importance du sous-enregistrement des décès d'enfants.

Celui-ci varie nettement suivant l'habitat (tableau 15). Il est particulièrement élevé dans la catégorie des familles mixtes, sans doute en raison des déplacements caractéristiques de ce groupe. De plus, les registres urbains sont davantage affectés que ceux des campagnes, peutêtre à cause de la mise en nourrice qui faisait décéder à l'extérieur de leur paroisse les enfants de la ville.

TABLEAU 15

Sous-enregistrement des décès d'enfants suivant le sexe et l'habitat

\begin{tabular}{lcccc}
\hline \multicolumn{1}{c}{ Sexe } & Urbain & Rural & Mixte & Ensemble \\
\hline Garçons & $35,4 \%$ & $22,1 \%$ & $41,0 \%$ & $28,7 \%$ \\
Filles & $26,0 \%$ & $16,8 \%$ & $50,0 \%$ & $26,8 \%$ \\
\hline Ensemble & $31,3 \%$ & $19,6 \%$ & $45,1 \%$ & $27,8 \%$ \\
\hline
\end{tabular}

En appliquant chacun de ces pourcentages à la catégorie qui lui est propre, on en arrive à rajouter 305 décès à ceux déjà connus d'enfants morts avant 15 ans; une telle correction représente pas moins de 55\% des 560 enfants sur lesquels nous nous interrogions. Ces décès ont ensuite été répartis proportionnellement aux décès enregistrés de chaque groupe d'âges.

On dispose donc maintenant de 1163 décès survenus avant 15 ans. Mais ces décès ne concernent que les enfants connus; or, nous l'avons vu dans l'étude de la fécondité, il existe des naissances qui sont inconnues pour de multiples raisons et il s'agit maintenant d'estimer dans quelle proportion ces inconnus sont décédés avant 15 ans. En se référant à des travaux antérieurs ${ }^{39}$, on est conduit à ajouter d'abord 118 décès pour tenir compte des lacunes relatives aux ondoyés décédés, puis 134 des 177 naissances inconnues à cause de la perte des registres, en prenant pour acquis que la mortalité de ces enfants suit la même distribution que celle des célibataires.

Au total, ce sont donc 557 décès que l'on ajoute aux 858 connus dès le départ, soit une correction de $39,4 \%$. La table de mortalité résultant de toutes ces corrections apparait au tableau 16.

\footnotetext{
39 Hubert Charbonneau, Vie et mort..., op. cit., 122-123.
} 


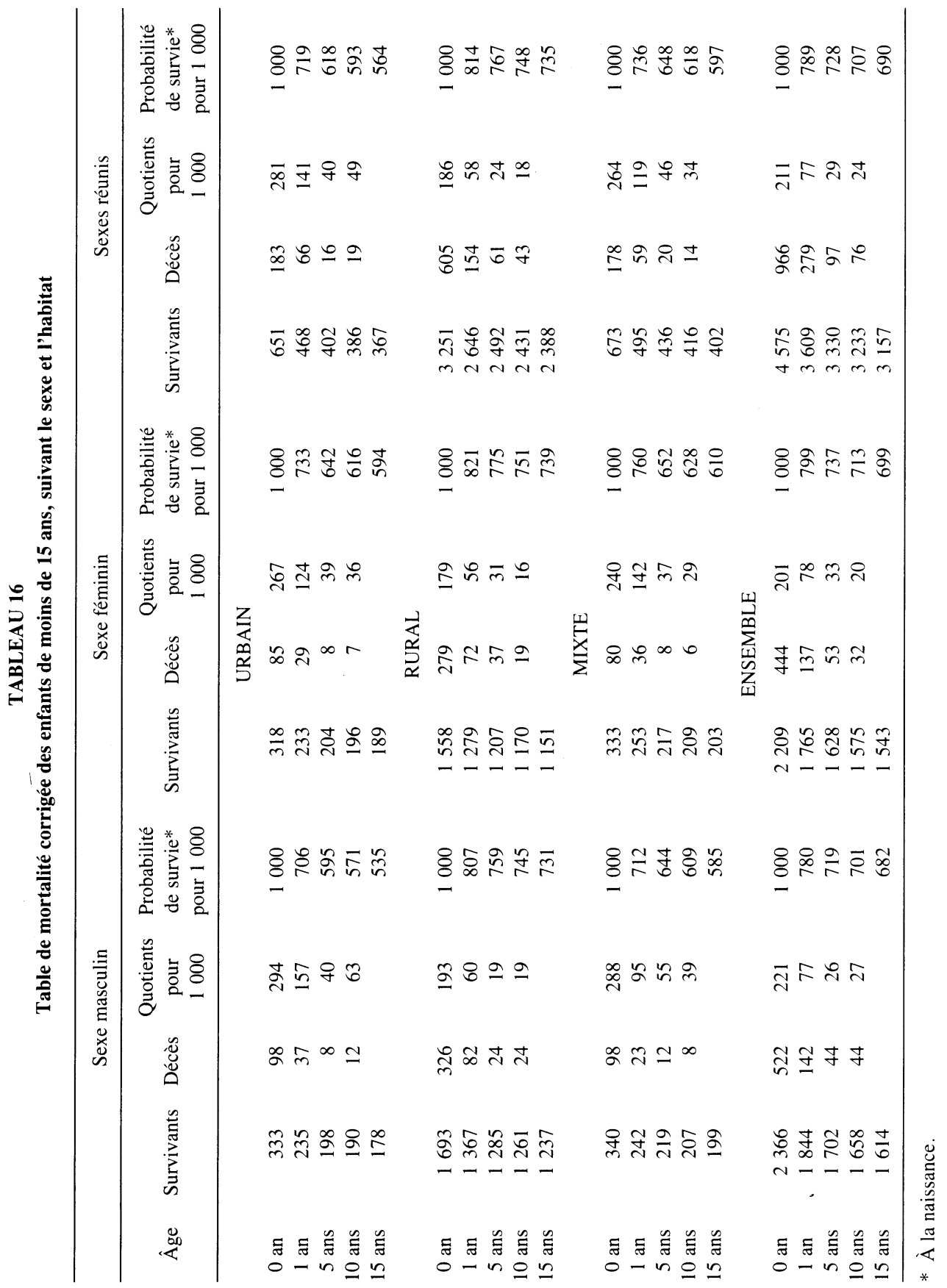


Cette table démontre d'abord que la mortalité infantile (avant 1 an) est nettement moins élevée à la campagne qu'à la ville ${ }^{40}$. La situation y reste d'ailleurs meilleure jusqu'à 15 ans, alors que la probabilité de survie à la campagne est de 735 pour 1000 et à la ville de seulement 564 pour 1000 . La situation des enfants de familles mixtes est intermédiaire, mais se rapproche beaucoup plus de celle de la ville que de celle de la campagne.

Si l'on décompose la mortalité infantile selon les facteurs endogènes (dépendant des conditions d'accouchement et des malformations de l'enfant) et exogènes (reliés aux conditions extérieures et à l'environnement), les écarts s'expliquent. En effet, le tableau 17 démontre qu'il n'y a pas de différence marquée dans la mortalité du premier mois selon qu'on habite à la ville ou à la campagne. Les familles mixtes sont cependant plus touchées, ce qui n'est peut-être pas étranger à leur ancienneté: on peut en effet supposer que les conditions d'accouchement étaient moins bonnes au début de la colonie et se sont améliorées avec le temps.

TABLEAU 17

Taux de mortalité endogène et exogène suivant l'habitat, sexes réunis (pour 1000 )

\begin{tabular}{lcccc}
\hline \multicolumn{1}{c}{ Mortalité } & Urbain & Rural & Mixte & Ensemble \\
\hline $\begin{array}{l}\text { Endogène } \\
\left(1^{\mathrm{er}} \text { mois }\right)\end{array}$ & 127 & 119 & 164 & 127 \\
$\begin{array}{l}\text { Exogène } \\
\left(2^{\mathrm{e}}-12^{\mathrm{e}} \text { mois }\right)\end{array}$ & 154 & 67 & 100 & 84 \\
\hline
\end{tabular}

En ce qui concerne la mortalité exogène, la campagne est nettement favorisée. Le taux y est très bas, surtout si on le compare à ceux déjà calculés pour la population rurale française ${ }^{41}$. Il faut donc voir la cause de cette mortalité infantile plus faible à la campagne uniquement dans les facteurs d'environnement comme l'hygiène, l'eau, la nourriture et la faible densité de peuplement, qui y étaient beaucoup plus favorables à la santé des jeunes enfants que dans les villes canadiennes. Tout porte à croire que celles-ci, malgré leur caractère embryonnaire à

40 Ainsi que l'avait déjà signalé Hubert Charbonneau, "À propos de démographie urbaine en Nouvelle-France - Réflexions en marge d'Habitants et marchands de Montréal auXVII siècle de Louise Dechêne», RHAF, 30, 2 (septembre 1976): 267.

41 Jacques Dupâquier, dans La population rurale du bassin parisien à l'époque de Louis XIV (Paris, 1979), 280, donne un taux de 156 pour mille. 
l'époque étudiée, portaient déjà en elles des conditions de vie assez défavorables pour diminuer la durée de vie des enfants et des adultes ${ }^{42}$.

\section{Conclusion}

Cette étude exploratoire des caractéristiques des populations urbaine et rurale de Nouvelle-France permet de constater que, comme pour la France, les habitants des villes se comportent différemment de ceux de la campagne.

Les écarts observés sont multiples. Certains sont dus au caractère même de la ville, plus anonyme que la campagne: ainsi s'expliquerait la fréquence plus élevée des conceptions prénuptiales dans les familles urbaines. D'autres sont dus au fait que la ville regroupe des familles de statut social plus élevé qu'à la campagne: la proportion élevée de célibataires et surtout de religieuses est un trait caractéristique des classes favorisées (surtout nobles) et l'âge au mariage plus élevé semble l'être également. D'autres différences relèvent de facteurs multiples: la mortalité des adultes plus forte à la ville implique une durée d'union plus courte qu'à la campagne ce qui, conjugué à l'âge au mariage déjà plus élevé, produit un nombre moyen d'enfants par famille plus faible. Par contre, le recours à la mise en nourrice, phénomène typiquement urbain attribuable à divers facteurs économiques et culturels, provoque, avec la mortalité infantile, une fécondité plus forte chez les couples des villes. Les conditions de l'environnement urbain jouent finalement un rôle prédominant dans la forte mortalité qui frappe les jeunes citadins.

Les facteurs d'explication d'ordre géographique semblent donc se conjuguer à d'autres facteurs liés aux caractéristiques sociales des groupes en présence. Une prochaine étape dans l'étude de la population de la Nouvelle-France consisterait donc à appréhender la population urbaine en la divisant en groupes sociaux, car l'image de la ville recouvre forcément des disparités importantes. Nos données sont ici trop restreintes pour effectuer cette répartition qui exige un nombre imposant de fiches de famille; cependant, le Programme de recherche en démographie historique de l'Université de Montréal a déjà en main tous les matériaux dont les chercheurs ont besoin pour continuer dans cette voie.

42 Cette différence dans la mortalité urbaine et rurale a été constatée par Alain Bideau, qui a comparé une ville française et sa campagne avoisinante, «La mortalité des enfants dans la châtellenie de Thoissey-en-Dombes - essai de pathologie historique (1670-1840)», dans Démographie urbaine $X V^{e}-X X^{e}$ siècle, op. cit.: 103-142. Malgré le peu de recherches comparatives formelles, elle est également admise pour l'ensemble de la France, Histoire de la France urbaine, tome 3 (Seuil, 1981): 51-53 et 344-345. 